\title{
Hybrid power quality conditioner for three-phase four-wire power systems
}

\author{
Maxim Chernyshov ${ }^{1}$, Valery Dovgun ${ }^{1}$, Sergei Temerbaev ${ }^{1, *}$, and Zumeyra Shakurova ${ }^{2}$ \\ ${ }^{1}$ Siberian Federal University, 79 Svobodny pr., Krasnoyarsk, 660041, Russia \\ ${ }^{2}$ Kazan State Power Engineering University,51 Krasnoselskaya street, Kazan, 420066, Republic of Tatarstan, Russia
}

\begin{abstract}
The article considers a hybrid power quality conditioner (HQPC) for 3-phase 4-wire systems with a distributed modular structure. Some conditioner modules provide compensation for the component currents and voltages that form the negative and zero sequence systems. The open structure of the HQPC, consisting of independent modules, allows compensating for distortions of currents and voltages of the 3phase network caused by the nonlinear nature and asymmetry of single-phase loads. The compensation characteristics of the proposed conditioner were researched using a model developed in the MatLab environment. The simulation showed that the proposed conditioner can ensure normalization of power quality in 3-phase 4-wire system at various modes of network operation.
\end{abstract}

\section{Introduction}

The issue of ensuring the power quality (PQ) in 3-phase 4-wire systems has become particularly important in recent years. The reason is the continuous increase of non-linear loads, such as energy-saving lighting systems, office and computer equipment.

Loads in low-voltage systems have a number of features that differ them from non-linear loads of industrial consumers. In contrast to industrial networks, where high-power loads are concentrated, office and commercial consumers are characterized by low-power single-phase non-linear loads, which current spectrum contains low-frequency 3,5 , and 7 th harmonics predominate. Harmonics that are multiples of three in 3phase networks form a zero-sequence system and are summed in neutral conductors.

Another feature of low-voltage four-wire network is the unequal distribution of single-phase loads. This causes phase current asymmetry, including higher harmonics asymmetry [1].

The widespread use of nonlinear single-phase loads has led to the fact that the asymmetry and non-sinusoidal nature of currents and voltages in 3-phase 4-wire systems have become permanent factors that reduce the efficiency of power supply systems $[1,2]$. It should also be taken into account that many consumers in such systems have increased requirements for power quality $[3,4]$. Asymmetry and non-sinusoidal nature of currents and voltages can lead to failures of control systems, failures in information transmission and processing systems. Therefore, the problem of creating filtercompensating devices for 3-phase 4-wire systems reducing the influence of the nonlinear nature and asymmetry of single-phase loads requires the new approaches.

Compensating devices for 3-phase 4-wire power systems are considered in [1-9]. In many cases, passive and hybrid compensating devices for 3-phase 4-wire systems are implemented on the basis of transformers with windings connected according to the "zigzag" scheme [1, 5-7]. However, the zigzag transformer also has a low impedance for zero-sequence components created by the external network. This can cause a significant increase the current in the neutral conductor if the network voltage is not symmetrical $[5,6]$.

The general task of PQ normalizing in 3-phase 4wire systems can be solved with the help of unified power quality conditioner (UPQC), proposed for the first time in [8]. The UPQC considered in [8,9] contains both series and parallel active filters. The disadvantage of UPQC is the high cost of the device. In many cases, a structure that includes both a serial and parallel active filter may be redundant

A more economical version of UPQC for 3-phase 4wire systems is proposed in the article [7]. It consists of two parallel hybrid filters that provide separate compensation for the symmetric components of the negative and zero sequence. The circuit solutions proposed in [7] allow reducing the power of active filters and thereby reducing the cost of the device.

Power quality conditioners, considered in [7 - 9], allow to solve the problems caused by harmonic distortion and unbalanced (asymmetric) load. However, these devices are designed to regulate PQ in network nodes that are connected to a concentrated non-linear load. For 3-phase 4-wire systems, in which the main share of the distorting load is made up of 1-phase low

* Corresponding author: s.a.temerbaev@ gmail.com 


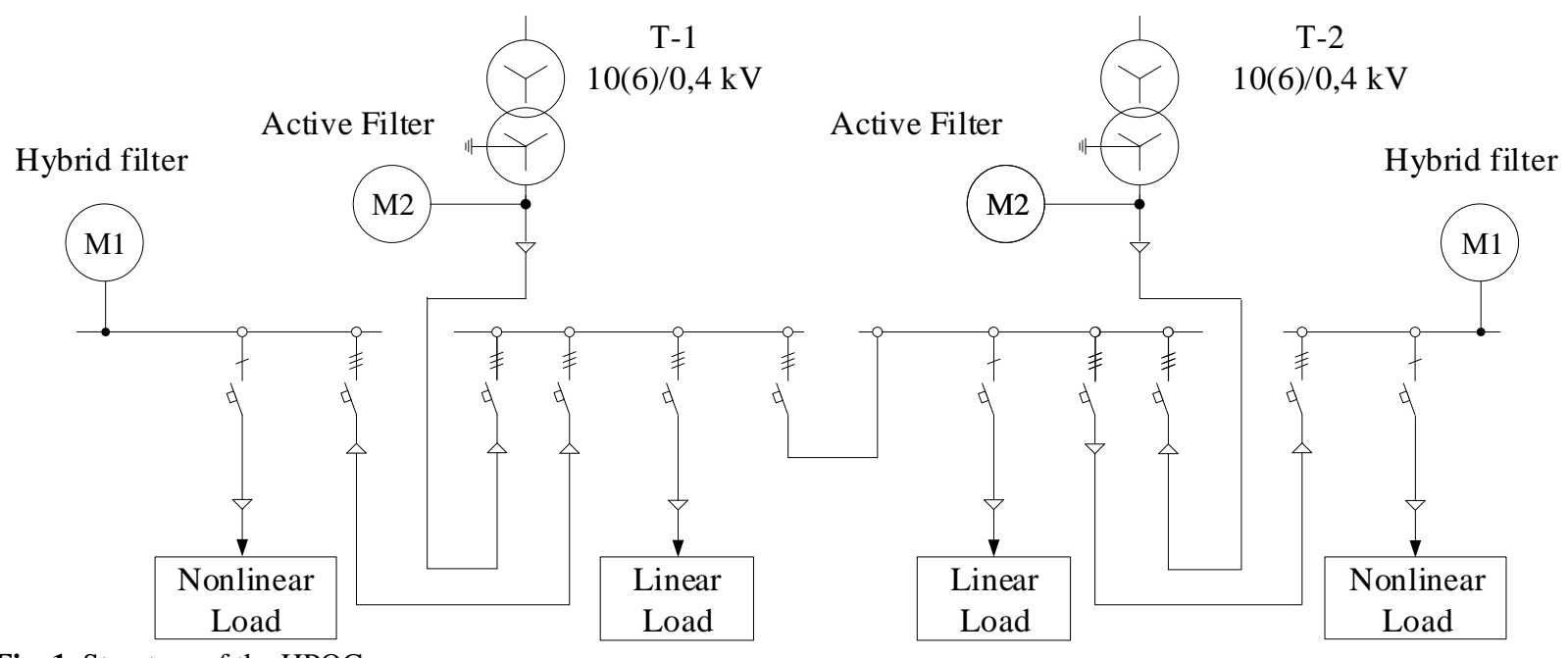

Fig. 1. Structure of the HPQC.

power receivers, it is necessary to use distributed power quality conditioners.

The article considers a hybrid power quality conditioner (HPQC) for 3-phase 4-wire systems with a distributed modular structure. The conditioner modules are installed in the network nodes to which a significant part of the nonlinear load is connected, and provide compensation for the currents and voltages components that form symmetrical systems of negative or zero sequence. A digital system for generating control signals for active filters that are part of the conditioner is considered. A model in the MatLab (Simulink) environment was developed to study the characteristics of HPQC.

\section{Configuration of a HPQC}

The structure of the proposed HPQC is shown in Fig. 1. The conditioner contains modules of filter compensating devices of two levels. First-level modules (M1), as shown in Fig.1, carry out the attenuation of the component currents and voltages that form a symmetrical system of zero sequence.

Since the majority of single-phase non-linear loads that generate zero-sequence currents are connected to network nodes inside the building, it is advisable to place M1 modules in such nodes.

Second-level modules (M2), as shown in Fig. 2, designed to compensate for components that form a symmetrical negative sequence system. They are installed in nodes that are connected to a load sensitive to reduced PQ, or they are connected to the secondary winding of a step-down transformer. This will reduce the impact of nonsymmetric and non-linear loads on the operating modes of consumers with high requirements for the PQ.

Let's consider the configuration of compensating devices that are part of the conditioner. The M1 module is a hybrid filter formed by a serial connection of a passive filter and a single-phase voltage inverter as shown in Fig. 2. The passive filter can be realized either in the form of resonant circuit tuned to the frequency of the dominant third harmonic, or in the form of a broadband passive filter that has low resistance at higher harmonic frequencies $(k=3,5, \ldots)$.

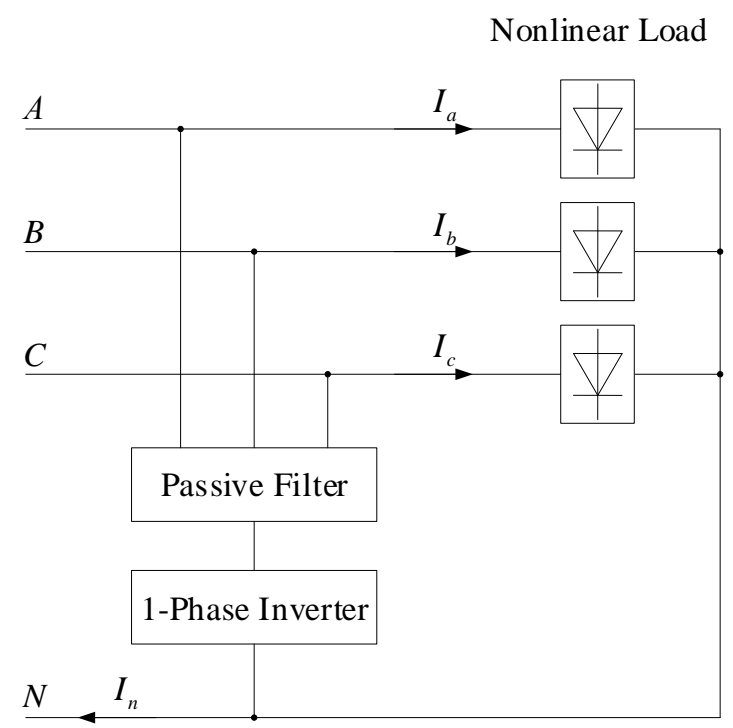

Fig. 2. Hybrid filter structure to reduce neutral wire current.

Single-phase inverter in the diagram in Fig. 2 act as an active filter. The inverter voltage is proportional to the harmonic components of the neutral conductor current:

$$
U_{A F}=R_{A F 1} I_{n}^{(1)}+R_{A F 2} I_{n}^{(h)}
$$

$R_{A F 1}, \quad R_{A F 2}$ are the control parameters of the active filter; $I_{n}^{(1)}$ is a component of the neutral conductor current with the fundamental frequency; $I_{n}^{(h)}$ are high-frequency components of the neutral current.

It was shown in [10] that at the frequency of the $k$-th harmonic, the current of the neutral conductor in the circuit as in Fig. 2.

$$
\&_{n}=\frac{3 E_{0 C}}{Z_{C}+Z_{P F}+3\left(Z_{N}+R_{A F}\right)}-\frac{Z_{P F} \&}{Z_{C}+Z_{P F}+3\left(Z_{N}+R_{A F}\right)}
$$


Voltage between neutral points

$$
\begin{aligned}
\mathscr{L}_{N n}^{\&} & =\frac{3 Z_{N} E_{0 C}}{Z_{C}+Z_{P F}+3\left(Z_{N}+R_{A F}\right)} \\
& -\frac{Z_{N} Z_{P F} \&}{Z_{C}+Z_{P F}+3\left(Z_{N}+R_{A F}\right)}
\end{aligned}
$$

$E_{0 \mathrm{C}}$ is a component of the power system voltage that forms a symmetric zero-sequence system; $J_{0 L}$ is zero sequence component of load current; $Z_{C}$ is the power system impedance; $Z_{P F}$ is the passive filter impedance; $Z_{N}$ is the impedance of the neutral wire.

From the formulas $(1,2)$, it follows that the action of the active filter is equivalent to turning on the resistance in series with the impedance of the neutral wire. This reduces the current in the neutral conductor and reduces the voltage between the neutral points. The hybrid filter also reduces the components of the neutral conductor current caused by the asymmetry and non-sinusoidal form of the external network voltage.

The M2 module is designed to compensate for the components that make up the negative sequence system. A parallel or serial hybrid filter can implement it. The M2 module in the form of a parallel hybrid filter is shown in Fig. 3.

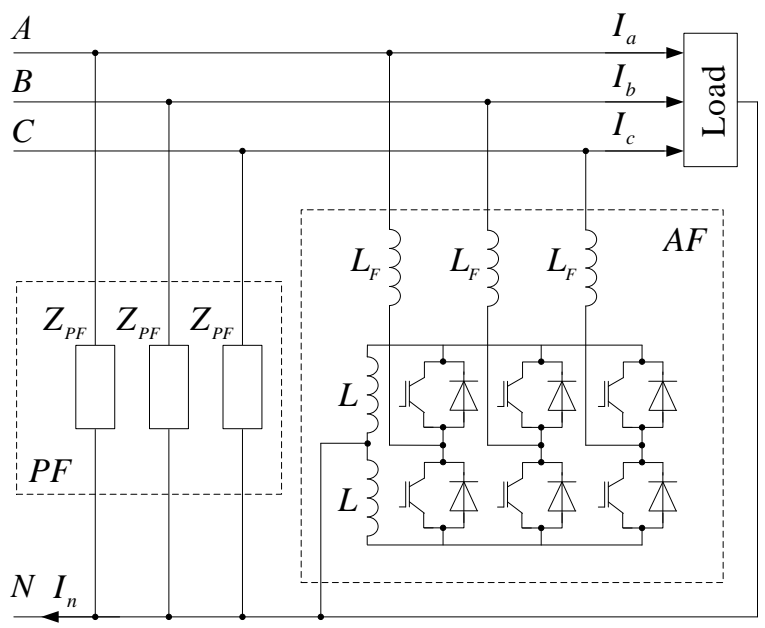

Fig. 3. Structure of a 3-phase hybrid filter.

The passive filter is tuned to the fifth harmonic. The active filter is implemented by a 3-phase 3-wire current inverter controlled by the voltage at the module connection point.

Active filter current $J_{A F}$ in the diagram in Fig. 3 is proportional to the voltage component at the point of common coupling, which forms the negative sequence system: $J_{A F}=G_{A F} U_{P C C}^{(2)}$. Voltage at the connection point of the nonlinear load and the hybrid filter can be expressed as

$$
U_{P C C}^{(2)}=\frac{Y_{C} E_{C}^{(2)}}{Z_{C}+Y_{P F}+G_{\text {aq }}}-\frac{J_{L}^{(2)}}{Z_{C}+Y_{P F}+G_{A F}}
$$

$E_{C}^{(2)}$ and $J_{L}^{(2)}$ are the components of voltage and current of the load forming system of negative sequence; $Y_{P F}$ is the conductivity of passive filter; $G_{A F}$ is the parameter of active filter, which has the conductivity dimension.

In accordance with (3), the active filter reduces the components of the negative sequence voltage at the point of common coupling (PCC) created by both the load and the external network. The reduction degree can be adjusted by changing the parameter of the active filter $G_{A F}$.

The proposed distributed structure of the HPQC is open and easy to modify, including new modules. Failure of one of the modules will not affect the efficiency of the conditioner in other nodes of the network. The distributed nature of the compensating system allows reducing the power of active filters. This reduces the cost of the system and increases its reliability.

\section{Digital system for generating control signals for active filters}

The proposed HPQC can be used as a multifunctional device that provides suppression of neutral conductor currents, compensation of voltage asymmetry in network nodes, and so on. Its characteristics depend on the methods used to generate control signals for active filters. In this case, it is advisable to use a strategy for generating control signals based on the use of instantaneous values of symmetrical components of voltages and currents of a 3-phase network [11-16].

The digital system for generating control signals for active filters consists of two parts. The first part (shown in Fig. 4) provides control of a 1-phase inverter that suppresses the current of the neutral conductor. It includes a frequency separation digital filter (FSDF), which generates signals proportional to the spectral components of the neutral current having the frequency of the main harmonic $i_{N}^{(1)}$ and high-frequency harmonics $i_{N}^{(h)}$, as well as a block for generating control signals for a 1-phase inverter.

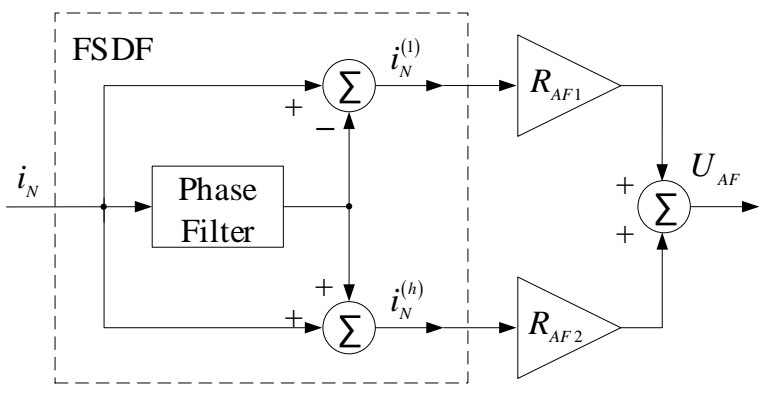

Fig. 4. Digital control signal generation system for 1-phase active filter.

The second-order phase filter in Fig. 4 has a transfer function whose module is equal to 1 in the entire frequency range. FSDF implements simultaneously the 
transfer functions of the band pass filter (BPF) and the band separating filter (BSF):

$$
\begin{aligned}
& H_{\mathrm{BSF}}(z)=\frac{i_{N}^{(h)}}{i_{N}}=\frac{1}{2}[1+A(z)] \\
& H_{B P F}(z)=\frac{i_{N}^{(1)}}{i_{N}}=\frac{1}{2}[1-A(z)]
\end{aligned}
$$

$A(z)$ is a transfer function of the phase filter.

The purpose of a 3-phase hybrid filter is to obtain fundamental phase voltages of the 3-phase system at the PCC, forming a positive sequence system. The remaining components of the phase stresses at the PCC caused by the asymmetry and non-linear nature of the loads must be reduced. To do this, in accordance with (3), the inverter voltages must be proportional to the components of the phase voltages to be compensated.

The block diagram of the 3-phase active filter control signal generation system is shown in Fig.5. Signals proportional to the line voltage of the network at the filter connection point are fed to the inputs of bandpass filters.

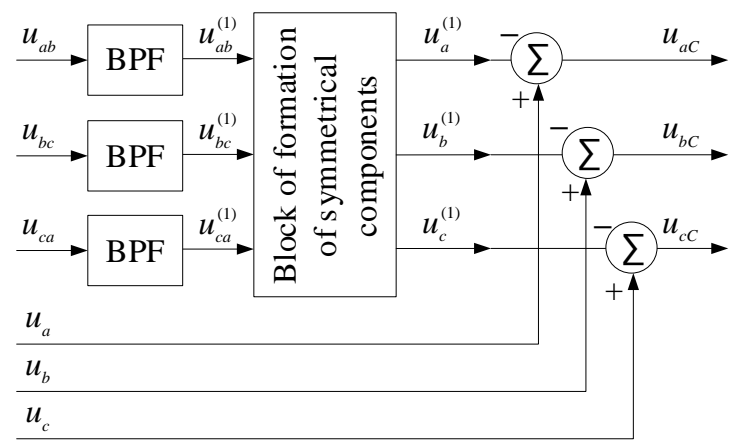

Fig. 5. Block diagram of the control signal generation system for a 3-phase active filter.
After selecting the components with the frequency of the main harmonic, the signals are fed to the input of the module for forming symmetrical components. The output signals of the module are the main frequency voltages that form a symmetrical system of direct sequence.

3-phase active filter control signals are defined by expressions:

$$
\begin{aligned}
& i_{a}=\frac{P}{\left(u_{a 1}^{(1)}\right)^{2}+\left(u_{b 1}^{(1)}\right)^{2}+\left(u_{c 1}^{(1)}\right)^{2}}\left(u_{a}-\left(u_{a 1}^{(1)}-u_{0}\right)\right) \\
& i_{b}=\frac{P}{\left(u_{a 1}^{(1)}\right)^{2}+\left(u_{b 1}^{(1)}\right)^{2}+\left(u_{c 1}^{(1)}\right)^{2}}\left(u_{b}-\left(u_{b 1}^{(1)}-u_{0}\right)\right) \\
& i_{c}=\frac{P}{\left(u_{a 1}^{(1)}\right)^{2}+\left(u_{b 1}^{(1)}\right)^{2}+\left(u_{c 1}^{(1)}\right)^{2}}\left(u_{c}-\left(u_{c 1}^{(1)}-u_{0}\right)\right)
\end{aligned}
$$

where $u_{0}=\frac{1}{3}\left(u_{a}+u_{b}+u_{c}\right), P=u_{a} i_{a}+u_{b} i_{b}+u_{c} i_{c} ; \quad u_{a 1}^{(1)}$, $u_{b 1}^{(1)}, u_{c 1}^{(1)}$ are the components of phase voltages of the main frequency that form a positive sequence system.

\section{HPQC modelling}

To study the characteristics of the proposed HPQC, a model was developed in the MatLab (Simulink) environment (Fig. 6). The M1 module is implemented with a passive broadband filter and a 1-phase inverter. A 3-phase active filter is used as the M2 module.

The model parameters are the following: the resistance of the network $R_{C}=0.4 \mathrm{Ohms}$, inductance $L_{C}=0.318 \mathrm{mH}$.

Three 1-phase bridge rectifiers represent symmetric nonlinear load in the diagram in Fig. 6. Its parameters

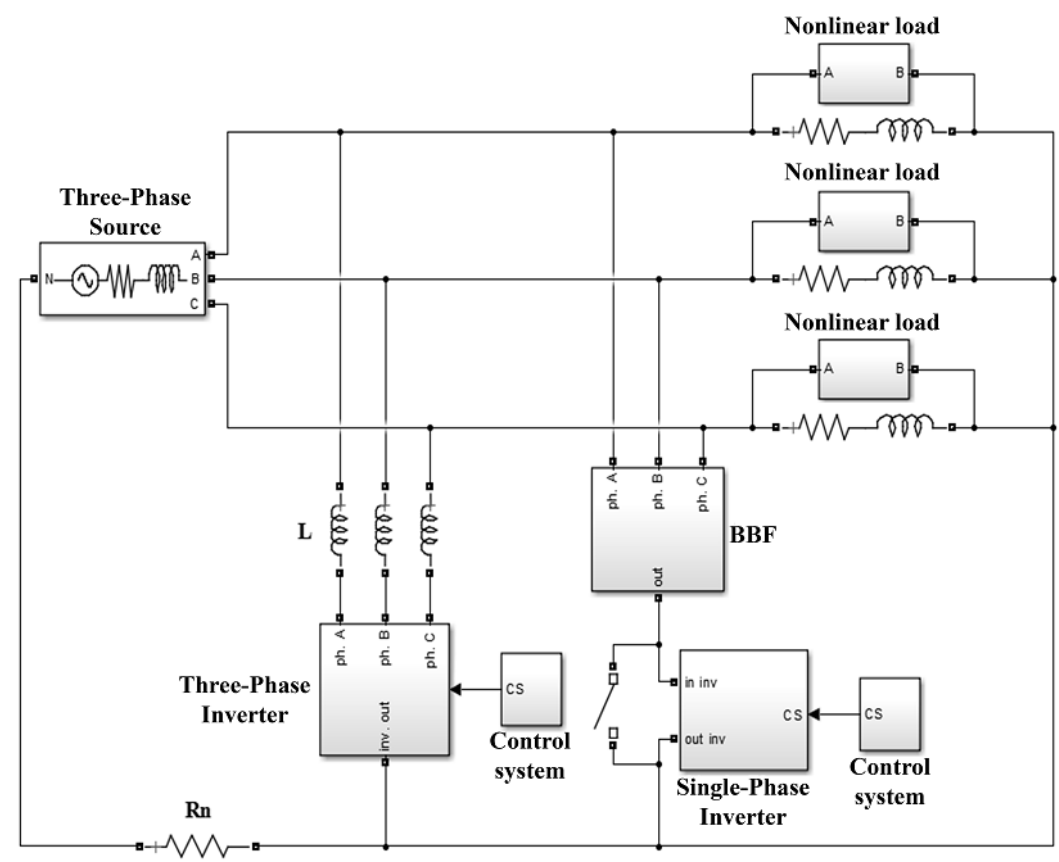

Fig. 6. MatLab Simulink model of HPQC. 
Table 1. Simulation results.

\begin{tabular}{|c|c|c|c|c|c|c|c|c|}
\hline Mode & $I_{a}, \mathrm{~A}$ & $I_{b}, \mathrm{~A}$ & $I_{c}, \mathrm{~A}$ & $I_{n}, \mathrm{~A}$ & $K_{2 U}, \%$ & $K_{U A}, \%$ & $K_{U B}, \%$ & $K_{U C}, \%$ \\
\hline Without filter-compensating devices & 29,71 & 16,5 & 16,9 & 17,9 & 5,3 & 9,5 & 8,4 & 8,4 \\
\hline With a zero sequence filter & 30,7 & 17,2 & 17,4 & 8,2 & 5,3 & 4,23 & 4,39 & 4,51 \\
\hline $\begin{array}{c}\text { Zero sequence filter + } \\
\text { negative sequence filter }\end{array}$ & 32,66 & 17,65 & 17,83 & 9,1 & 1,1 & 1,91 & 1,87 & 1,85 \\
\hline
\end{tabular}

are the following: resistance $R_{L}=40 \mathrm{Ohms}$, smoothing filter capacity $C=937.5 \mu \mathrm{F}$.

Parameters of an asymmetric linear load in phase $A$, the load resistance $R_{L}=5$ Ohms, inductance $L_{L}=15.9 \mathrm{mH}$, in phases $B$ and $C$, the load resistance $R_{L}=15 \mathrm{Ohms}$, inductance $L_{L}=47.8 \mathrm{mH}$.

The results of the simulation are presented in Table 1.

The simulation results show that after switching on the conditioner, the current of the neutral wire has reduced almost twice. The voltage asymmetry coefficient for the negative sequence $K_{2 U}$ has reduced by more than three times and is within the norm defined by GOST standard 32144-2013. Distortion of phase currents and voltages has also been significantly reduced.

\section{Conclusion}

The article considers a HQPC for 3-phase 4-wire systems that provides compensation for current and voltage distortions caused by the nonlinear nature and asymmetry of single-phase loads. There are proposed configurations of modules that compensate the components forming symmetric systems of negative and zero sequence.

A mathematical model of the proposed HQPC in the MatLab environment is developed. The model is used to study the compensatory characteristics of the HQPC. Analysis of the simulation results showed that the use of the proposed device can normalize the power quality in 3-phase 4-wire systems under different operating modes. With a physical model it is planned to conduct a detailed analysis of the capabilities of the conditioner.

\section{References}

[1] A.K. Shidlovsky, A.F. Zharkin, Higher Harmonics in Low Voltage Electrical Networks $(210,2005)$

[2] T. Key, J-S Lai, Analysis of harmonic mitigation methods for building wiring systems, IEEE Transactions on Power Systems 13, 3, 890 (1998)

[3] J. Pomilio, S. Deckmann, Characterization and compensation of harmonics and reactive power of residential and commercial loads, IEEE Transactions on Power Delivery 22, 2, 1049 (2007)

[4] H. Hafezi, G. D’Antona, A. Dede, D.D. Giustina, R. Faranda, G. Massa, Power quality conditioning in LV distribution networks: results by field demonstration, IEEE Transactions on Smart Grid, 8, 1, 418 (2017)

[5] H.L. Jou, J.C. Wu, K.D. Wu, W.J. Chiang, Y.H. Chen, Analysis of zig-zag transformer applying in the three-phase four-wire distribution power system, IEEE Transactions on Power Delivery 20, 2, 1168 (2005)

[6] B. Singh, P. Jayaprakash, T. Somayajulu, D. Kothari, Reduced rating VSC with zig-zag transformer for current compensation in a threephase four-wire distribution system, IEEE Transactions on Power Delivery 24, 1, 249 (2009)

[7] J.C. Wu, H.L. Jou, H.H. Hsiao, S.T. Xiao, A new hybrid power conditioner for suppressing harmonics and neutral-line current in three-phase four-wire distribution power systems, IEEE Transactions on Power Delivery 29, 4, 1525 (2014)

[8] H. Fujita, H. Akagi, The unified power quality conditioner: The integration of series- and shuntactive filters, IEEE Transactions on Power Electronics 13, 2, 315 (1998)

[9] V. Khadkikar, Enhancing power quality using UPQC: a comprehensive overview, IEEE Transactions on Power Electronics 27, 5, 2284 (2012)

[10] V.P. Dovgun, M.O. Chernyshov, O.E. Maloshenok, Hybrid power filters for three-phase four-wire networks, News of Higher Educational Institutions, Energy issues 1-2, 11 (2016)

[11] K. Uyyuru, M. Mishra, A. Ghosh, An optimization-based algorithm for shunt active filter under distorted supply voltages, IEEE Transactions on Power Electronics 24, 5, 1223 (2009)

[12] E.I. Gracheva, O.V. Naumov, Evaluation criteria of contact group technical state concerning electrical appliances, International Journal of pharmacy and Technology 4, 27084-27091 (2016)

[13] D.D. Micu, I.V. Ivshin, E.I. Gracheva, O.V. Naumov, A.N. Gorlov, Influence of resistance of contact units of switching devices on the electric power losses in shop networks of low tension, SES-2019, E3S Web of Conferences 124, 02013, 1-4 (2019)

[14] E.I. Gracheva, V.N. Petrov, N.V. Russova, D.V. Samuilov, G.P. Svintsov, I.V. Protosovitski, On modeling on a single-winding forced valve 
electromagnet in a circuit with a ballast resistor, SES-2019, E3S Web of Conferences 124, 02012, 5-9 (2019).

[15] E. Gracheva, A. Alimova, Calculation Methods and Comparative Analysis of Losses of Active and Electric Energy in Low Voltage Devices, 2019 International Ural Conference on Electrical Power Engineering (UralCon), Chelyabinsk, Russia, 361-367 (2019)

[16] A.N. Egorov, Ya.S. Kharitonov, V.M. Khubieva, I.A. Yakushev, N.V. Golubtsov, Z.M. Shakurova, The impact of higher voltage and current harmonics on the operation of electrical systems, SES-2019, E3S Web of Conferences 124, 05067, (2019) 